\title{
Interpretations of and management actions following electrocardiograms in symptomatic patients in primary care: a retrospective dossier study
}

\author{
L. M. E. Wagenvoort · R. T. A. Willemsen · K. T. S. Konings · H. E. J. H. Stoffers
}

Published online: 12 July 2019

(c) The Author(s) 2019

\begin{abstract}
Background The electrocardiogram (ECG) has become a popular tool in primary care. The clinical value of the ECG depends on the appropriateness of the indication and the interpretation skills of the general practitioner (GP).

Objectives To describe the use of electrocardiography in primary care and to assess the performance of GPs in interpreting ECGs and making subsequent management decisions.

Methods Three hundred ECGs, recorded during daily practice in symptomatic patients by 14 GPs who regularly perform electrocardiography, were selected. Corresponding data of the indications, interpretations and subsequent management actions were extracted from the associated medical records. A panel consisting of an expert GP and a cardiologist reviewed the ECGs and specified their agreement with the findings and actions of the study GPs.

Results The most common indications were suspicion of a rhythm abnormality (43.7\%), ischaemic heart disease $(42.7 \%)$ and patient reassurance $(14.3 \%)$. The study GPs interpreted $53.3 \%$ of the ECGs as showing no (new or acute) abnormality, whereas supraventricular rhythm disorders $(12.3 \%)$, conduction disorders $(7.7 \%)$ and repolarisation disorders $(7.0 \%)$ were the most frequently reported pathological findings. Over-
\end{abstract}

Electronic supplementary material The online version of this article (https://doi.org/10.1007/s12471-019-01306-y) contains supplementary material, which is available to authorized users.

L. M. E. Wagenvoort · R. T. A. Willemsen (ه) •

K. T. S. Konings · H. E. J. H. Stoffers

Care and Public Health Research Institute (CAPHRI),

Department of Family Medicine, Maastricht University,

Maastricht, The Netherlands

robert.willemsen@maastrichtuniversity.nl all, the expert panel disagreed with the interpretations of the study GPs in $16.2 \%$ of cases, and with the GPs' management actions in $11.7 \%$. Learning goals for GPs performing electrocardiography could be formulated for acute coronary syndrome, rhythm disorders, pulmonary embolism, reassurance, left ventricular hypertrophy and premature ventricular complexes. Conclusion GPs who feel competent in electrocardiography performed well in the opinion of the expert panel. We formulated various learning objectives for GPs performing electrocardiography.

\section{What's new?}

- Common everyday indications to perform an electrocardiogram (ECG) in primary care are: suspicion of a rhythm abnormality, ischaemic heart disease and reassurance of the patient.

- Half of all ECGs recorded by general practitioners (GPs) revealed no (new or acute) abnormality. Frequent pathological findings were supraventricular rhythm disorders, conduction disorders and repolarisation disorders.

- Overall, GPs who feel competent in electrocardiography performed well in the opinion of the expert panel. However, the expert panel disagreed with $16.2 \%$ of the GPs' ECG interpretations and $11.7 \%$ of the GPs' management actions. The panel disagreed with both the interpretation and the subsequent management action in $5 \%$ of cases.

- Learning goals for GPs performing electrocardiography could be formulated for acute coronary syndrome, rhythm disorders, pulmonary embolism, reassurance, left ventricular hypertrophy and premature ventricular complexes. 
Keywords Electrocardiography · General practice · Quality of health care · Clinical skills · Diagnosis . Cross-sectional studies

\section{Introduction}

\section{General background}

The electrocardiogram (ECG) has become a frequently used and effective tool in primary care [1-3]. Nonetheless, the value of the ECG in primary care has been a recurrent topic of debate [1, 4-7]. The clinical value of the ECG strongly depends on the appropriateness of the indication and the competence of the physician interpreting the ECG [1, 2, 8-13]. Studies on the competence of general practitioners (GPs) have shown varying results [8, 13-16]. Competence is demanded since the quality of computer interpretation of ECGs is insufficient [17-20]. Besides, although Dutch primary care guidelines advocate the use of ECGs in specific situations (www.nhg.org/nhgstandaarden), clear guidelines on the use of ECGs in primary care settings are not available [21-23]. Currently, the actual performance regarding indications for and interpretation of the ECG among GPs is unknown.

To learn more about the use and usefulness of ECGs in primary care, we conducted a series of four studies (www.nhg.org/onderzoeken/het-ecg-denederlandse-huisartspraktijk-0). We expect these will support future ECG training for GPs. The first study addressed the competence of GPs in requesting and interpreting ECGs by means of a case vignette study [24]. In the remaining three studies, we have focussed on the performance of GPs in various real-life situations: ECGs recorded during out-of-office hours (to be published), ECGs performed during primary care cardiovascular risk management programmes (to be published) and ECGs carried out in symptomatic patients during day-care practice (this study).

\section{Objective}

The objective of this study was to describe the use of everyday ECGs recorded for symptomatic patients in primary care and to assess the performance of GPs in interpreting ECGs and making subsequent management decisions.

\section{Methods}

\section{Setting and design}

Between September and October 2015, 14 GPs who regularly record and interpret ECGs themselves were recruited at an ECG training programme or by e-mail for this retrospective dossier study. All ECGs that had been performed and interpreted by the GPs during office hours following a complaint reported to the GP or a finding observed during physical examination ('symptomatic') were analysed. An expert panel reviewed the interpretations of all ECGs and subsequent management actions.

\section{ECG data}

A master's student of medicine (L.M.E. Wagenvoort) performed this study during a compulsory science elective of 18 weeks. In the practices of the participating GPs (Fig. 1), she reviewed the practice ECG archive and included ECGs performed during office hours in patients with certain symptoms or physical examination findings ('symptomatic'). We excluded ECGs recorded in the context of cardiovascular risk management ('screening ECGs') or requested by other health care professionals. Patient characteristics and data on ECG indication, interpretation by the GP and
Fig. 1 Participating practices, general practitioners (GPS) and collection of electrocardiograms (ECGs). We included ECGs that had been recorded and interpreted by the GPs during office hours in symptomatic patients, i.e. after a complaint reported to the GP or a finding observed during physical examination. Routinely performed ECGs for screening purposes were excluded

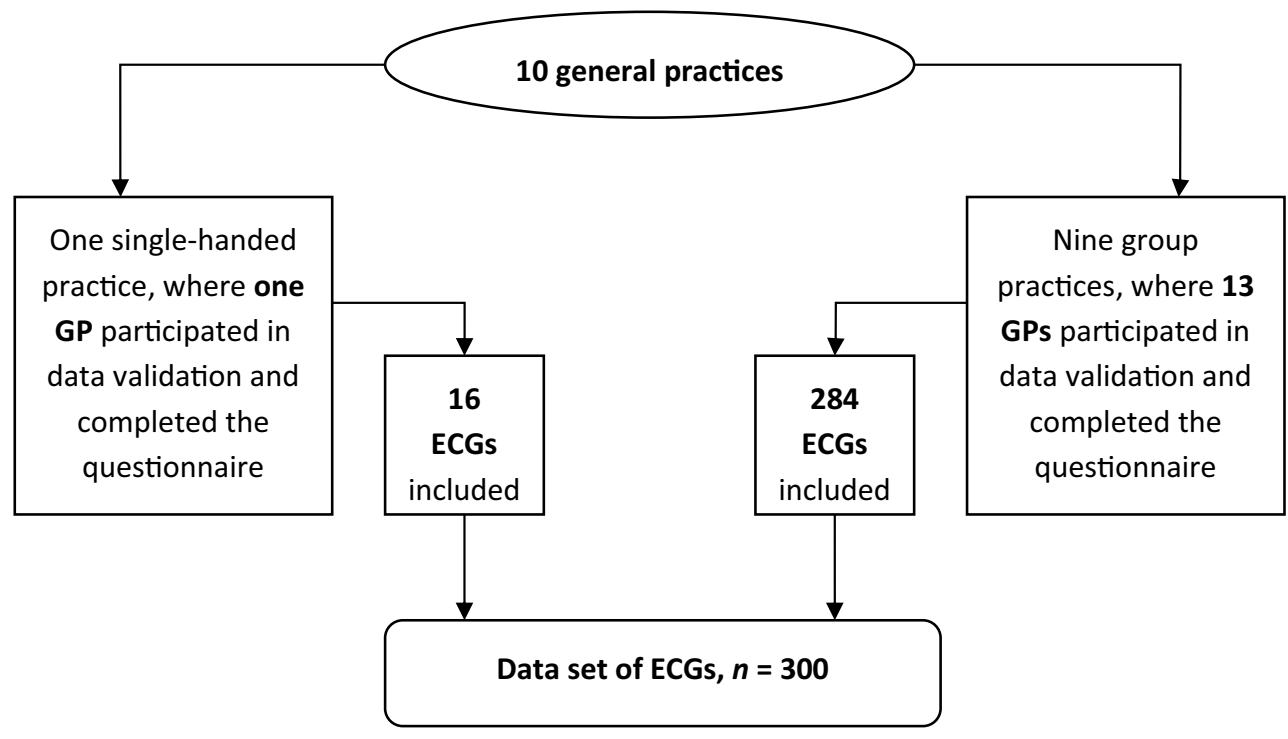


Fig. 2 Percentage of presenting symptoms and signs in patients in whom the GP recorded an ECG, as reported in the medical records $(n=300)$. More than one symptom or sign may have been mentioned per ECG. 'Other $(n=7)$ ' included: heart enlargement on chest radiography, biliary colic pain, panic attack, electrolyte abnormality, undefined, neurological deficit (2x) (ECG electrocardiogram, GP general practitioner)

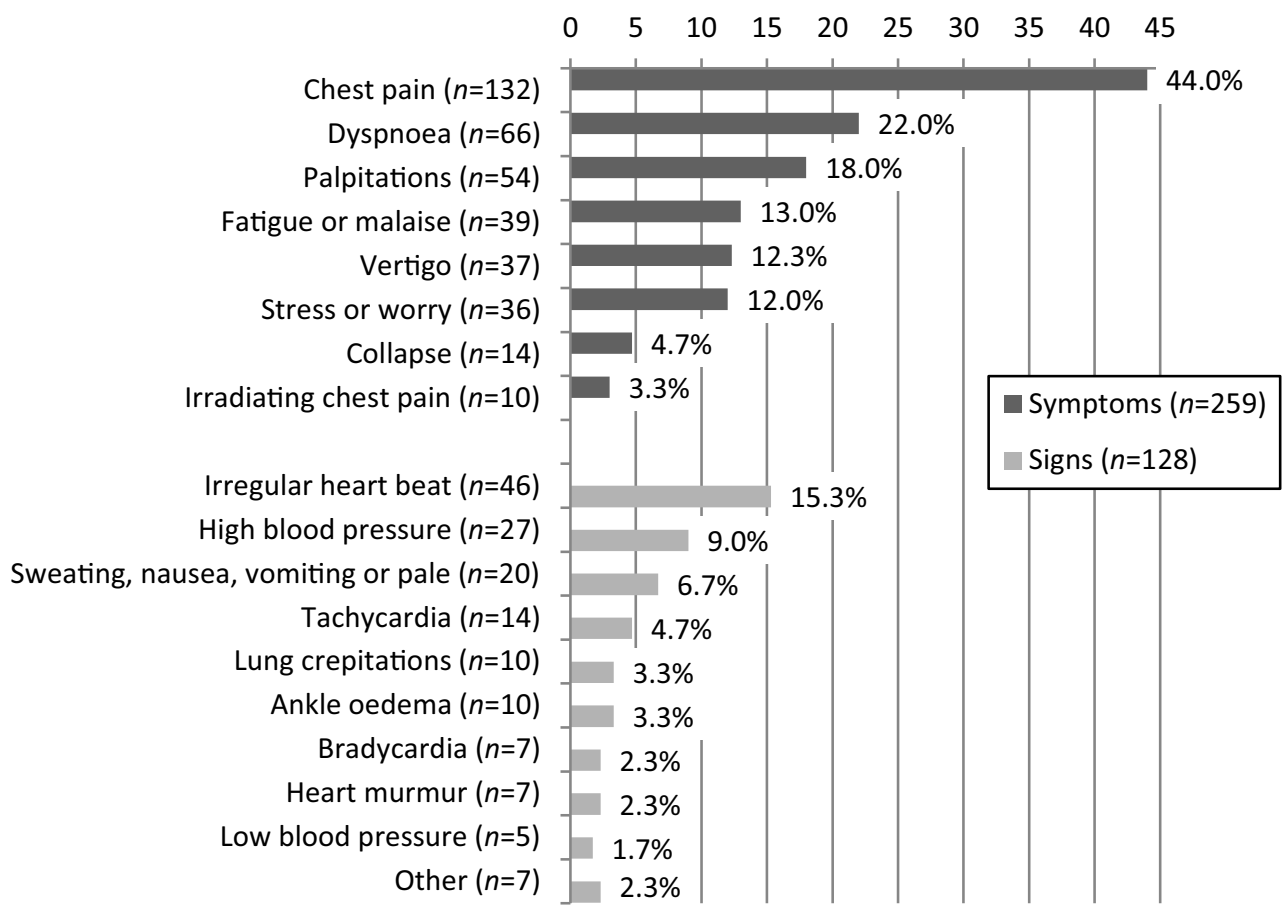

subsequent management actions were traced in the electronic medical record system and copied to an anonymised case record form. Any uncertainties were clarified in a dialogue with the GP. Each GP also completed a short questionnaire on personal ECG skills (for an overview of all data, see Supplementary Table 1).

\section{Expert panel}

Two highly experienced GPs and four cardiologists were included in the expert panel. Both expert GPs had worked as a cardiology resident for several years and successfully completed an acknowledged 2-year training course on cardiovascular disease. For each ECG, one expert GP and one cardiologist reviewed interpretations and management actions. In the first round, the panel interpreted the ECG and accompanying data, blinded for the interpretation of the study GP. Then, the interpretation of the GP was revealed, and the experts indicated and explained the motivation for their (dis)agreement with the performance of the GP. If there was a difference in opinion between the two panel members, the second expert GP was consulted and determined the final panel verdict.

\section{Statistics}

Descriptive analyses were made of GP characteristics, patient characteristics, ECG indications, interpretations and management actions, and the panel assessment using the Statistical Package for the Social Sciences (SPSS) version 21.

\section{Ethical considerations}

This retrospective dossier study was not subject to the Dutch 'Medical Research Involving Human Subjects Act' (WMO). Case record forms did not contain personal data; all study data were anonymous. Any relevant disagreement of interpretation between the panel and the study GP was expected to become clinically irrelevant 3 months after the date of the

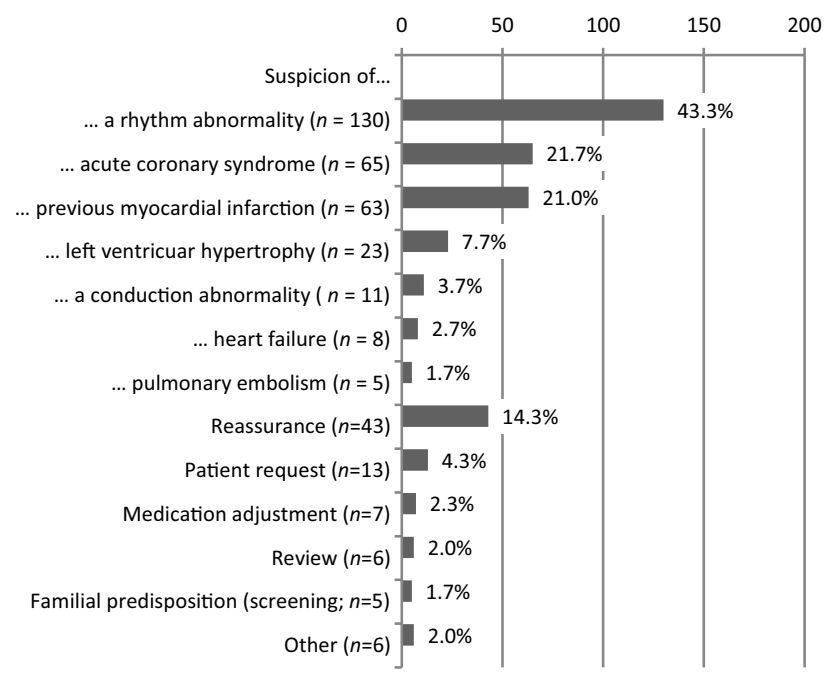

Fig. 3 Indications for ECGs by GPs (percentage of all ECGs, $n=300$ ). Since occasionally more than one indication was reported, the total number of reported indications $(n=385)$ exceeds the number of ECGs $(n=300)$. 'Other $(n=6)$ ' included: myocarditis, pericarditis, hyperkalaemia, suspected long QT interval, aneurysm of abdominal aorta, no indication. All mentioned once (ECG electrocardiogram, GP general practitioner) 
Table 1 Frequencies of ECG interpretations reported by the GP

\begin{tabular}{|c|c|c|}
\hline GP's ECG interpretation & $n$ & Percentage of all ECGs $(n=300)$ \\
\hline No (acute or new) abnormalities & 163 & 54.3 \\
\hline - Normal & 121 & 40.3 \\
\hline - No changes compared to previous ECG & 19 & 6.3 \\
\hline - No acute pathology & 23 & 7.7 \\
\hline Sinus node arrhythmia & 17 & 5.7 \\
\hline - Sinus arrhythmia & 8 & 2.7 \\
\hline - Sinus tachycardia & 5 & 1.7 \\
\hline - Sinus bradycardia & 4 & 1.3 \\
\hline Supraventricular arrhythmia & 36 & 12.0 \\
\hline - Atrial fibrillation & 30 & 10.0 \\
\hline - Atrial flutter & 4 & 1.3 \\
\hline - Ectopic atrial rhythm & 1 & 0.3 \\
\hline - Premature supraventricular complex & 1 & 0.3 \\
\hline Premature ventricular complex & 14 & 4.7 \\
\hline Conduction abnormality & 28 & 9.3 \\
\hline - First-degree AV block & 4 & 1.3 \\
\hline - Second-degree AV block & 1 & 0.3 \\
\hline - Ventricular pre-excitation (Wolff-Parkinson-White pattern) & 1 & 0.3 \\
\hline - Nodal rhythm & 2 & 0.7 \\
\hline - Right bundle branch block & 10 & 3.3 \\
\hline - Left bundle branch block & 6 & 2.0 \\
\hline - Left anterior fascicular block & 3 & 1.0 \\
\hline - Trifascicular block & 1 & 0.3 \\
\hline QRS axis deviation & 14 & 4.7 \\
\hline - Left axis deviation & 13 & 4.3 \\
\hline - Right axis deviation & 1 & 0.3 \\
\hline Repolarisation abnormalities & 21 & 7.0 \\
\hline - Non-specific ST/T abnormality & 8 & 2.7 \\
\hline - ST/T abnormality suggestive of acute ischaemia & 13 & 4.3 \\
\hline Abnormalities suggestive of old myocardial infarction & 18 & 6.0 \\
\hline - Non-acute signs of myocardial ischaemia & 9 & 3.0 \\
\hline - Slow R progression & 3 & 1.0 \\
\hline - Pathological Q wave(s) & 6 & 2.0 \\
\hline Left ventricular hypertrophy & 6 & 2.0 \\
\hline Abnormal, not specified & 3 & 1.0 \\
\hline ECG interpretation of the study GP missing & 38 & 12.7 \\
\hline - Missing & 28 & 9.3 \\
\hline - ECG interpreted by cardiologist & 10 & 3.3 \\
\hline
\end{tabular}

visit. Therefore, we only included ECGs that had been recorded more than 3 months before the date of the actual data collection.

\section{Results}

\section{Characteristics of GPs and ECGs}

Details of the 14 participating GPs are shown in Supplementary Table 2. The median number of years of experience as a GP was 17 years. Four GPs had worked in a cardiology department in the past, 12 GPs had completed an ECG course. The reported median number of interpreted ECGs per month was 14.

Altogether, the ECGs of 300 individual patients were included (Fig. 1). The mean age of the patients was 61 years (range 14-92), 44.3\% (133/300) were male, and in $82 \%(245 / 300)$ at least one cardiovascular risk factor or cardiovascular disease had been identified previously (see Supplementary Table 3 for more details). 
Table 2 Frequencies of management actions taken by the GP after the ECG $(n=300)$

\begin{tabular}{|c|c|c|}
\hline \multirow{4}{*}{$\begin{array}{l}\text { No specialist } \\
\text { involved } \\
(n=187)\end{array}$} & No action & $130(43.3 \%)$ \\
\hline & Further diagnostic evaluation by GP & $39(13 \%)$ \\
\hline & Medication adjustment by GP & $13(4.3 \%)$ \\
\hline & Medication and further diagnostic evaluation by GP & $5(1.7 \%)$ \\
\hline \multirow{6}{*}{$\begin{array}{l}\text { Specialist } \\
\text { involved } \\
(n=113)\end{array}$} & Further diagnostic evaluation and routine referral to cardiologist & $2(0.7 \%)$ \\
\hline & Medication and routine referral to cardiologist & $1(0.3 \%)$ \\
\hline & Routine referral to cardiologist & $27(9 \%)$ \\
\hline & $\begin{array}{l}\text { Telephone consultation with cardiologist (followed by medication adjustment } 6 \text {, further diagnostic examination in primary } \\
\text { care } 4 \text {, both medication adjustment and further examination } 1 \text {, referral } 2 \text { ) }\end{array}$ & $29(9.7 \%)$ \\
\hline & Immediate referral to cardiologist & $42(14 \%)$ \\
\hline & Referral to other specialist & $12(4 \%)$ \\
\hline
\end{tabular}

\section{ECG indications}

In Fig. 2, the signs and symptoms of patients in whom an ECG was performed are presented, and in Fig. 3 the indications for the ECGs are listed. The most frequently reported indications were (suspicion of) a rhythm abnormality (43.3\%), an acute coronary syndrome $(21.7 \%)$ or an unknown old myocardial infarction $(21.0 \%)$. Patient 'reassurance' (14.3\%) was another commonly reported indication.

\section{ECG interpretations}

All ECG interpretations as documented by the GPs are listed in Tab. 1. The GPs interpreted 54.3\% (163/300) of the ECGs as showing 'no (new or acute) abnormality'. Supraventricular arrhythmia was reported in 36 $(12.0 \%)$ cases. In $28(9.3 \%)$ cases no interpretation could be extracted from the patient record, and in 10 (3.3\%) cases the GP had requested the cardiologist to interpret the ECG.

\section{Management actions following the ECG}

Management actions by the GP following the ECG are listed in Tab. 2. In 130 of 300 (43.3\%) cases no further action was taken. In most cases, actions following the ECG were taken by the GP alone (187/300, 62.3\%). In 113 of 300 cases $(37.7 \%)$ a specialist, mainly a cardiologist, was involved. Among these were 42 cases of immediate referral to a cardiologist, 30 routine referrals and 29 cases of consultation by telephone/telefax (of whom two patients were referred subsequently).

When the GP interpreted the ECGs as showing 'no (new) abnormality' ( $n=160)$, no further management action was taken in $63 \%(100 / 160)$ of cases, and further diagnostic evaluation was planned in $14 \%$ (23/160). In 43 ECG cases for which the indication was 'reassurance', $86 \%$ of the ECGs were followed by 'no action'. When the indication was 'suspicion of acute coronary syndrome', the GP referred $42 \%$ of the patients (27/65) immediately in. In nine of these 27 referred cases, the ECG showed no abnormal findings.
For the most frequently reported symptom preceding the ECG, namely 'chest pain' $(n=132)$, the most frequent subsequent actions were 'no action' in 53 cases $(40 \%)$, and 'immediate referral' in 29 cases $(22 \%)$, including nine cases in which the ECG revealed no abnormalities.

\section{Comparison to expert panel}

Due to missing GP interpretations ( $n=28$ ) or poor ECG quality $(n=7)$, the panel could not review the GP's interpretation of 35 of the 300 ECGs (12\%). There was full agreement (ECG interpretation plus management) between the expert panel and GPs on 207 of the $300(69 \%)$ cases. In 43 of the $265(16.2 \%)$ assessable ECG interpretations, the panel disagreed on one or more aspects of the ECG interpretation of the study GP (Fig. 4). The panel disagreed with the GP's man-

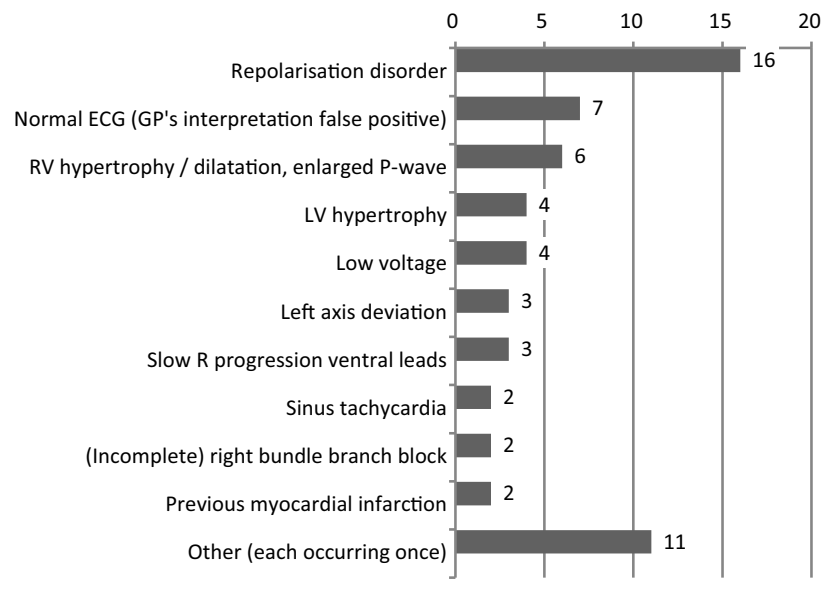

Fig. 4 Absolute numbers of ECG abnormalities missed or incorrectly interpreted by GPs. In 43 out of 300 ECGs, 60 missed or incorrectly interpreted ECG abnormalities were described by the expert panel. 'Other $(n=11)$ ' included: sinus bradycardia, supraventricular premature complex, atrial flutter, nonsustained ventricular tachycardia, 1st degree AV block, 3rd degree AV block, right axis deviation, horizontal axis, 'pathological' Q-wave, S1Q3 pattern, lead reversal. All mentioned once (AV atrioventricular, ECG electrocardiogram, GP general practitioner, $L V$ left ventricular, $R V$ right ventricular) 
Table 3 Learning goals derived from the expert panel's observations in this study to improve GPs' competence in interpreting ECGs recorded in primary care patients

\begin{abstract}
Observation
Several patients with chest pain were referred immediately although the electrocardiogram (ECG) was normal. This is in accordance with guidelines stating that an ECG is not suitable to rule out acute coronary syndrome (ACS) in acute situations [1]. However, several chest pain patients with normal ECG findings were not referred immediately
\end{abstract}

In one case, the expert panel disagreed on the GP's exclusion of a rhythm disorder based on a negative ECG, which was recorded at a time when the patient was not experiencing the reported complaints

In one ECG, the indication was 'suspicion of pulmonary embolism'. Since the study GP interpreted this ECG as 'normal' and no management action followed, it appeared that the study GP used the ECG to exclude pulmonary embolism A normal diagnostic test does not necessary reassure patients [25]. Therefore, using an ECG for reassurance can be regarded as doubtful. However, the expert panel considered reassurance to be an important part of a GP's work, leading to a high level of agreement on ECGs performed to reassure patients

The expert panel considered the indication 'left ventricular hypertrophy' (LVH) often to be unfounded, since hypertension should be treated properly irrespective of the presence of LVH

In one ECG, the GP interpreted the series of broad QRS complexes as multiple premature ventricular complexes (PVCs), whereas the expert panel described this ECG as non-sustained ventricular tachycardia

\section{Learning goal}

Although the causal relationship between the normal findings on the ECG and the subsequent non-referral is difficult to establish, it seems reasonable to conclude that when teaching interpretation of ECGs to general practitioners (GPS), one learning goal should be that ECGs are unsuitable to rule out ACS in acute chest pain cases

Especially for confirming or excluding rhythm disorders, an ECG should be recorded when the symptoms are being experienced

The negative predictive value of such an ECG is too low, leading to the conclusion that exclusion of pulmonary embolism is not possible based on an ECG

Thus, reassurance seems feasible. However as pointed out earlier, the negative predictive value of an ECG in ruling out rhythm disorders in the absence of symptoms, or ACS, is low

The indication 'left ventricular hypertrophy' is doubtful

Although PVCs are usually innocent in primary care, three or more PVCs in a row, as well as fusiform or multiform PVCs, should be viewed with caution. Referral to a cardiologist for further risk assessment of ventricular rhythm disorders is necessary agement action in 35 of the 300 (11.7\%) cases; in 15 of these cases (5\% of all ECGs) the panel disagreed on the interpretation as well as the management.

In most cases in which they disagreed with the GP's management, the panel advised further examination, medication adjustment or referral to the outpatient cardiology clinic. However, in two cases, contrary to the GP's original decision, the panel advised making an immediate referral: one case of suspected acute coronary syndrome where the panel agreed that the ECG revealed no relevant abnormalities, and one case of bradycardia that had been missed by the GP.

\section{Discussion}

\section{Main findings}

In this study of 300 ECGs recorded during daily practice in symptomatic patients by 14 GPs, the most frequently reported indications were (suspicion of) a rhythm abnormality, ischaemic heart disease and patient 'reassurance'. In $86 \%$ of the latter ECG cases, the ECG was not followed by any action. The GPs interpreted half of the ECGs as revealing no (new or acute) abnormality. Supraventricular rhythm disorders, conduction disorders and repolarisation disorders were the most frequently reported pathological findings.

In around $60 \%$ of the analysed cases, the ECG was followed by either no further action, a medication change or an additional diagnostic test. In almost $40 \%$ of cases, a cardiologist was consulted immediately or at a later time. In 27 of 65 cases of 'suspected acute coronary syndrome', the GP referred immediately, including 9 cases without an ECG abnormality.
The panel disagreed with the GPs' interpretations in $16.2 \%$ of the assessable ECGs. The panel disagreed with the GPs' management actions in $11.7 \%$ of all ECG cases. In most of the latter cases, the panel would have advised further examination, medication adjustment or referral to the outpatient cardiology clinic. However, in two cases, contrary to the GP's original decision, the panel would have advised referring immediately: one case of suspected acute coronary syndrome with a normal ECG and one case of (missed) bradycardia.

\section{ECGs performed in symptomatic patients by GPs during office hours}

In $84 \%$ of the assessable ECGs, the panel agreed with the interpretation of the study GP. This result is similar to those of other studies, in which agreement between the GPs and a cardiologist was found in $80-90 \%$ of cases $[4,13]$. The panel entirely disagreed with (both the interpretation and the subsequent management action of) the study GP in 5\% of cases. However, management actions may have been postponed in some cases. The rather low percentage of disagreement on interpretation and management may be regarded as an indication that GPs master electrocardiography in everyday symptomatic cases satisfactorily.

However, from the points of disagreement between GP and expert panel, learning goals for GPs performing electrocardiography could be formulated for acute coronary syndrome, rhythm disorders, pulmonary embolism, reassurance, left ventricular hypertrophy and premature ventricular complexes (Tab. 3). In addition, our previous vignette study on the competence of GPs in requesting and interpreting ECGs 
revealed that GPs showed poor diagnostic accuracy for left anterior fascicular block and incomplete right bundle branch block [24].

The precise role of ECGs in the clinical reasoning of the GP, the potential of eHealth collaboration between GP and cardiologist, and possibly machine learning in interpreting ECGs outside the cardiology practice may be topics for future research.

\section{Strengths and limitations}

To our knowledge, our study is the first to provide insight into indications for, interpretations of, and management actions following ECGs recorded in everyday symptomatic patients in primary care. We analysed a rather large sample of 300 real-life ECGs performed and interpreted by GPs who do this routinely. Therefore, we regard our results to be representative for ECGs recorded by GPs who feel competent in electrocardiography. Since we analysed historical data, the behaviour of the participating GPs was not influenced by the study. We collected as much clinical information as possible to enable the expert panel to assess each ECG in its clinical context. Our panel assessment was based on the independent judgement of at least two panel members, a cardiologist and a GP. The panel disagreed with the management action only if there were clear reasons for this disagreement.

Some limitations need to be acknowledged. We described the use of electrocardiography in primary care and had a panel judge the ECGs that were performed as they were. However, we were not able to judge the usefulness of electrocardiography in primary care, since the decisions to perform electrocardiography had already been made and correlations with management decisions were not assessable due to the retrospective design of the study. Our results can only be generalised to GPs who feel sufficiently competent in interpreting ECGs. The quality of the extracted data depended on the documentation in the medical records, although we clarified uncertainties in a conversation with the GP. To assess whether abnormalities were new and thus more relevant was only possible in a minority of cases where previous ECGs were available. The members of the panel judged the cases only on paper, and may have missed factors that were decisive for the participating GPs, such as the complete clinical picture, including background knowledge of the patient. Furthermore, the panel had no access to previous ECGs. Moreover, due to the pragmatic study design, the panel was unable to determine the precise connection between the study GP's ECG interpretation and the subsequent management action. In this light, the (dis)agreement of the panel with the GPs' decisions is to a certain extent arbitrary. Several patient factors can contribute to a management action that seems erroneous when assessing the case on paper, but may be defendable in a real-life context. Finally, since we did not have any follow-up data on the pa- tients, we do not know whether the disagreements on management actions were actually indicative of adverse clinical outcomes.

\section{Conclusions}

This study indicates that GPs who feel competent in electrocardiography perform satisfactorily with regard to both the interpretation of ECGs recorded in symptomatic patients and to subsequent management actions. To further improve the ability of GPs to interpret ECGs, we have formulated various learning objectives for GPs performing electrocardiography.

Acknowledgements The authors would like to thank all participating GPs for their collaboration and hospitality; and N.H.T. Dinh, Professor A.P.M. Gorgels, P. Luyten and O.L. Reuchlin, cardiologists, for their generous and competent contributions to the expert panel.

Funding This work was not supported by any external funding. At the time the study was performed, L.M.E. Wagenvoort was participating in a research elective for medical students at Maastricht University during the final year of her master's degree.

\section{Compliance with ethical guidelines}

Conflict of interest L.M.E. Wagenvoort, R.T.A. Willemsen, K.T.S. Konings and H.E.J.H. Stoffers declare that they have no competing interests.

Ethical standards This retrospective dossier study was not subject to the Dutch 'Medical Research Involving Human Subjects Act' (WMO).

Open Access This article is distributed under the terms of the Creative Commons Attribution 4.0 International License (http://creativecommons.org/licenses/by/4.0/), which permits unrestricted use, distribution, and reproduction in any medium, provided you give appropriate credit to the original author(s) and the source, provide a link to the Creative Commons license, and indicate if changes were made.

\section{References}

1. Chan CM, Willemsen RTA, Konings KTS. Electrocardiographyin general practice. Huisarts Wet. 2014;57:196-200.

2. Konings K, Willemsen RTA, Bertholet GJM. Evaluating and understanding ECGs. The. ECG, Vol. 10. method. Houten: BohnStafleu van Loghum; 2017.

3. Dubin D. Snelle interpretatie van ECG's (vierde herziene druk, based on 6th revised edition of Rapid interpretation of EKG's). Amsterdam: Reed Business Education; 2013.

4. Rutten FH, Kessels AG, Willems FF, Hoes AW. Electrocardiography in primary care; is it useful? Int J Cardiol. 2000;74:199-205.

5. De Ruijter W. ECGs in general practice: milk cow or Trojan horse? Huisarts Wet. IEEE Trans Med Imaging. 2014;57:404.

6. Scheltens T, De Beus MF, Hoes AW, et al. The potential yield of ECG screening of hypertensive patients: the Utrecht Health Project. J Hypertens. 2010;28:1527-33.

7. Margolis S, Reed R. EKG analysis skills of family practice residents in the United Arab Emirates: a comparison with US data. Fam Med. 2001;33:447-52. 
8. Jensen MS, Thomsen JL, Jensen SE, Lauritzen T, Engberg M. Electrocardiogram interpretation in general practice. Fam Pract. 2005;22:109-13.

9. Zwietering P, KnottnerusA, Gorgels T, RinkensP.Occurrence of arrhythmias in general practice. Scand J Prim Health Care. 1996;14:244-50.

10. Houghton AR, Sparrow NJ, Toms E, Cowley AJ. Should general practitioners use the electrocardiogram to select patients with suspected heartfailure for echocardiography? Int JCardiol. 1997;62:31-6.

11. Bogun F, Anh D, Kalahasty G, et al. Misdiagnosis of atrial fibrillation and its clinical consequences. Am J Med. 2004;117:636-42.

12. White T, Woodmansey P, Ferguson DG, Channer KS. Improving the interpretation of electrocardiographs in an accident and emergency department. Postgrad Med J. 1995;71:132-5.

13. Santos P, Pessanha P, Viana M, et al. Accuracy of general practitioners' readings of ECG in primary care. Cent Eur J Med. 2014;9:431-6.

14. Macallan DC, Bell JA, Braddick M, Endersby K, RizzoNaudi J. The electrocardiogram in general practice: its use and its interpretation. JR Soc Med. 1990;83:559-62.

15. Mant J, Fitzmaurice DA, Hobbs FD, et al. Accuracy of diagnosing atrial fibrillation on electrocardiogram by primary care practitioners and interpretative diagnostic software: analysis of data from screening for atrial fibrillation in the elderly (SAFE) trial. BMJ. 2007;335:380.

16. Whitman M, LaytD, Yelland M. Key findings on ECGs-level of agreement between GPs and cardiologists. Aust Fam Physician. 2012;41:59-62.

17. Hwan Bae M, Hoon LJ, Heon YD, et al. Erroneous computer electrocardiogram interpretation of atrial fibrillation and its clinical consequences. Clin Cardiol. 2012;35:348-53.

18. Mawri S, Michaels A, Gibbs J, et al. The comparison of physician to computer interpreted electrocardiograms on
ST-elevation myocardial infarction door-to-balloon times. Crit Pathw Cardiol. 2016;15:22-5.

19. Schlapfer J, Wellens HJ. Computer-interpreted electrocardiograms: benefits and limitations. J Am Coll Cardiol. 2017;70:1183-92.

20. Hughes KE, Lewis SM, Katz L, Jones J. Safety of computer interpretation of normal triage electrocardiograms. Acad Emerg Med. 2017;24:120-4.

21. Santos P, Martins C, Sa L, Hespanhol AP, Couto L. Motives for requesting an electrocardiogram in primary health care. Cien Saude Colet. IEEE Trans Med Imaging. 2015;20:1549:54.

22. Sur DK, Kaye L, Mikus M, Goad J, Morena A. Accuracy of electrocardiogram reading by family practice residents. Fam Med. 2000;32:315-9.

23. Surawicz B, Childers R, Deal BJ, Gettes LS. AHA/ACCF/HRS Recommendations for the standardization and interpretation of the electrocardiogram: Part III: Intraventricular conduction disturbances. A scientific statement from the American Heart Association Electrocardiography and Arrhythmias Committee, Council on Clinical Cardiology; the American College of Cardiology Foundation; and the Heart Rhythm Society endorsed by the International Society for Computerized Electrocardiology. J Am Coll Cardiol. 2009;53:976-81.

24. Compiet SAM, Willemsen RTA, Konings KTS, Stoffers H. Competence of general practitioners in requesting and interpreting ECGs-a case vignette study. Neth Heart J. 2018;26:377-84.

25. Rolfe A, Burton C. Reassurance after diagnostic testing with a low pretest probability of serious disease: systematic review and meta-analysis. Jama Intern Med. 2013;173:407-16. 